\title{
Endoplasmic reticulum stress preconditioning antagonizes low-density lipoprotein-induced inflammation in human mesangial cells through upregulation of XBP1 and suppression of the IRE1 $\alpha / \mathrm{IKK} / \mathrm{NF}-\kappa \mathrm{B}$ pathway
}

\author{
YUAN YU ${ }^{1}$, LING ZHANG ${ }^{1}$, QI LIU ${ }^{2}$, LIN TANG ${ }^{1}, \mathrm{HANG} \mathrm{SUN}^{2}$ and HUI GUO ${ }^{2}$ \\ ${ }^{1}$ Department of Nephrology; ${ }^{2}$ Institute for Viral Hepatitis, Key Laboratory of Molecular Biology for Infectious Diseases, \\ The Second Affiliated Hospital, Chongqing Medical University, Chongqing 400010, P.R. China
}

Received December 25, 2013; Accepted September 18, 2014

DOI: $10.3892 / \mathrm{mmr} .2014 .2960$

\begin{abstract}
Elevated plasma low-density lipoprotein (LDL) is associated with systemic inflammation, and is an important factor in the pathogenesis of chronic kidney disease. The aim of the present study was to investigate the effects of endoplasmic reticulum (ER) stress preconditioning on LDL-induced inflammatory responses, in human mesangial cells (HMCs). HMCs were exposed to LDL (200 nm), with or without pretreatment with tunicamycin, an ER stress inducer, and tested for changes to gene expression levels. Small interfering RNA technology was used to knockdown the expression of inositol-requiring enzyme-1 $\alpha$ (IRE1 $\alpha$ ) and X-box-binding protein-1 (XBP-1), in order to determine their effects on LDL-treated HMCs. LDL treatment resulted in a significant, and time-dependent, increase in the relative mRNA expression levels of proinflammatory cytokines and CD40, which was coupled with enhanced phosphorylation of IRE1 $\alpha$, I $\mathrm{B}$ kinase (IKK), and nuclear factor (NF)- $\mathrm{B}$ p 65 and p65 nuclear translocation. The LDL-induced inflammatory responses were significantly reduced in the IRE1 $\alpha$-depleted HMCs. Furthermore, pretreatment with tunicamycin significantly attenuated the induction of proinflammatory cytokines and CD40, by LDL. Whereas, silencing XBP1 expression significantly restored the production of proinflammatory cytokines, in the LDL-treated HMCs with ER stress preconditioning. The phosphorylation levels of IRE1 $\alpha$, IKK, and NF- $\kappa$ B p65 were markedly increased in the XBP1-depleted HMCs. Conversely, overexpression of XBP1 blocked LDL-induced inflammation in the HMCs. The results of the present study demonstrate that ER stress preconditioning antagonizes LDL-induced inflammatory responses
\end{abstract}

Correspondence to: Dr Ling Zhang, Department of Nephrology, The Second Affiliated Hospital, Chongqing Medical University, 76 Linjiang Lu, Chongqing 400010, P.R. China

E-mail: lindazhang8508@hotmail.com

Key words: chronic kidney disease, endoplasmic reticulum stress, feedback regulation, inflammation, protective effects in HMCs, which may be mediated through upregulation of $\mathrm{XBP} 1$, and subsequent inactivation of the IRE1 $\alpha / \mathrm{IKK} / \mathrm{NF}-\kappa \mathrm{B}$ pathway.

\section{Introduction}

Chronic kidney disease (CKD) is a growing health problem worldwide (1), which manifests as a progressive loss of renal function. Numerous factors, including oxidative stress, systemic inflammation, hypertension, and dyslipidemia, contribute to the onset and progression of CKD (2). Lipid abnormalities in patients with CKD usually consist of reduced high-density lipoprotein (HDL) concentrations and elevated plasma triglyceride, low-density lipoprotein (LDL), and oxidized lipids and lipoproteins (3). HDL facilitates cholesterol efflux from peripheral tissues and delivers cholesterol to the liver for excretion, therefore it has a key role in reverse cholesterol transport (4). HDL confers protection from cardiovascular disease due to its high anti-oxidant and anti-inflammatory activities. LDL has converse roles to HDL in the regulation of cholesterol transport. Oxidized LDL is capable of inducing inflammatory responses and is therefore implicated in the progression of numerous inflammatory disorders, such as atherosclerosis (5) and CKD (3).

The endoplasmic reticulum (ER) is a central organelle of eukaryotic cells and has principle functions in lipid synthesis and protein folding, maturation, and transport. Disturbances to the normal functions of the ER (e.g. due to redox dysregulation, aberrant calcium regulation, and glucose deprivation) may result in the accumulation of unfolded or misfolded proteins in the ER, a state known as ER stress (6). Three major ER stress response transducers have previously been identified: Activating transcription factor-6, inositol-requiring enzyme-1 $\alpha$ (IRE1 $\alpha$ ), and protein kinase RNA-like endoplasmic reticulum kinase (7). IRE1 $\alpha$ is activated by homodimerization and autophosphorylation under ER stress. It functions as an endoribonuclease which splices X-box-binding protein-1 (XBP-1) mRNA, yielding a potent transcriptional activator. Splicing of XBP-1 has previously been shown to initiate the transcription of genes involved in protein folding, transport and ER-associated protein degradation (7). 
ER stress initially serves as an adaptive response but may lead to cell death, if severe or prolonged ER dysfunction occurs. Previous research has demonstrated the importance of ER stress in the pathophysiology of both acute and chronic kidney diseases (8). Chiang et al (9) reported that overwhelming ER stress may induce renal cell apoptosis and subsequent fibrosis. However, numerous studies have recently supported a protective role of the induction of ER stress in cell damage (10-12). Li et al (10) reported that ER stress preconditioning mitigated retinal endothelial inflammation, which was mediated through activation of the XBP-1-mediated unfolded protein response (UPR) and inhibition of $N F-\kappa B$ signaling. In a rat model, preconditioning with ER stress ameliorated mesangioproliferative glomerulonephritis (12). These previous findings suggest that transiently targeting ER stress may have therapeutic benefits in the treatment of inflammatory diseases.

Inflammation is an important mechanism leading to glomerular damage in CKD (13). Native and modified LDL have previously been shown to increase the production of numerous inflammatory mediators, including interleukin- 6 (IL-6), CD40, and macrophage migration inhibitory factor, in human mesangial cells (HMCs) (14). The present study aimed to investigate whether ER stress preconditioning could attenuate LDL-induced inflammatory responses in HMCs, and to explore the associated molecular mechanisms.

\section{Materials and methods}

Chemical reagents and antibodies. LDL was purchased from ProSpec (East Brunswick, NJ, USA) and tunicamycin from Sigma-Aldrich (St. Louis, MO, USA). Mouse anti-human monoclonal antibodies anti-XBP1 and anti- $\beta$-actin were purchased from Santa Cruz Biotechnology, Inc. (Dallas, TX, USA), anti-phospho-NF-кB p65 (Ser536), and anti-phospho-I $\kappa$ B kinase (IKK) $\alpha / \beta$ (Ser176/180) were obtained from Cell Signaling Technology, Inc. (Danvers, MA, USA), and anti-phospho-IRE1 $\alpha$ and anti-IRE1 $\alpha$ were purchased from Novus Biologicals (Littleton, CO., USA).

Cell culture and drug treatment. Primary HMCs were obtained from the Key Laboratory of Infectious Diseases of Chongqing Medical University (Chongqing, China). The cells were cultured in Dulbecco's Modified Eagle's Medium, supplemented with $10 \%$ fetal bovine serum, $100 \mu \mathrm{g} / \mathrm{ml}$ streptomycin, and $100 \mathrm{U} / \mathrm{ml}$ penicillin (Invitrogen Life Technologies, Carlsbad, CA, USA), at $37^{\circ} \mathrm{C}$ in an atmosphere containing $5 \% \mathrm{CO}_{2}$. The experiments were performed with cells taken from passages 3-8.

The HMCs were treated with LDL $(200 \mathrm{~nm})$ for various durations (1-24 h), and then subjected to gene expression analysis. To assess the effects of ER stress preconditioning on the LDL-induced inflammatory responses, the cells were exposed for $8 \mathrm{~h}$ to a low dose $(0.1 \mu \mathrm{g} / \mathrm{ml})$ of tunicamycin, an ER stress inducer, followed by treatment with LDL.

Plasmids, small interfering RNA (siRNA) oligonucleotides, and transfection. The full-length human XBP1 cDNA was amplified and subcloned into an expression vector pEGFP-C1 (ClonTech, Palo Alto, CA, USA), which encoded a C-terminal green fluorescent protein tag. Specific siRNAs targeting
Table I. Sequences of the primers for RT-qPCR analysis.

\begin{tabular}{|c|c|}
\hline Gene & Sequence $\left(5^{\prime}-3^{\prime}\right)$ \\
\hline IL-6 & $\begin{array}{l}\text { Forward: TGCAATAACCACCCCTGACC } \\
\text { Reverse: ATTTGCCGAAGAGCCCTCAG }\end{array}$ \\
\hline TNF- $\alpha$ & $\begin{array}{l}\text { Forward: CTTCTCGAACCCCGAGTGAC } \\
\text { Reverse: TGAGGTACAGGCCCTCTGAT }\end{array}$ \\
\hline CD40 & $\begin{array}{l}\text { Forward: ACCCTTGGACAAGCTGTGAG } \\
\text { Reverse: GGCAAACAGGATCCCGAAGA }\end{array}$ \\
\hline MCP-1 & $\begin{array}{l}\text { Forward: AGCAGCAAGTGTCCCAAAGA } \\
\text { Reverse: TTTGCTTGTCCAGGTGGTCC }\end{array}$ \\
\hline XBP1 & $\begin{array}{l}\text { Forward: CAGTTGTCACCCCTCCAGAA } \\
\text { Reverse: GGCTGGTAAGGAACTGGGTC }\end{array}$ \\
\hline IRE $1 \alpha$ & $\begin{array}{l}\text { Forward: AAAACTACGCCTCCCCTGTG } \\
\text { Reverse: GTCAGATAGCGCAGGGTCTC }\end{array}$ \\
\hline GAPDH & $\begin{array}{l}\text { Forward: CAATGACCCCTTCATTGACC } \\
\text { Reverse: GACAAGCTTCCCGTTCTCAG }\end{array}$ \\
\hline
\end{tabular}

RT-qPCR, reverse transcription-quantitative polymerase chain reaction; IL-6, interleukin-6; TNF- $\alpha$, tumor necrosis factor- $\alpha$; MCP-1, monocyte chemoattractant protein-1; XBP1, X-box binding protein 1; IRE1 $\alpha$, inositol-requiring enzyme $1 \alpha$.

human IRE1 $\alpha$ and XBP1 were synthesized by Invitrogen Life Technologies, with the following sense sequences: IRE1 $\alpha, 5$ '-GUCCCACUUUGUGUCCAAUTT-3'; and XBP1, 5'-CCAAGCUGGAAGCCAUUAATT-3'. A scrambled siRNA sequence, 5'-UUCUCCGAACGUGUCACGUTT-3', was used as a negative control. The final concentration of each siRNA was $2 \mu \mathrm{M}$. The HMCs were seeded in 6-well plates, at a density of $5 \times 10^{4}$ cells/well, $24 \mathrm{~h}$ prior to transfection. The siRNA molecules were transfected into the cells using Lipofectamine ${ }^{\circledR} 2000$ reagent, according to the manufacturer's instructions (Invitrogen Life Technologies). The transfection efficiency was determined by western blot analysis of the corresponding protein levels, $24 \mathrm{~h}$ following siRNA transfection.

Quantitative polymerase chain reaction ( $q P C R)$. Total cellular RNA was extracted using the RNeasy kit, according to the manufacturer's instructions (Qiagen, Hilden, Germany). Reverse transcription was performed using the AMV First Strand cDNA Synthesis kit (Bio Basic Inc., Amhurst, NY, USA). The qPCR was conducted using an Applied Biosystems StepOnePlus Real-Time PCR System (Applied Biosystems, Foster City, CA, USA) and the SYBR ${ }^{\circledR}$ green PCR master mix (Applied Biosystems). GAPDH was amplified in a parallel reaction, and was used as an internal quantitative control. The cycle threshold $(\mathrm{Ct})$ was calculated for each gene tested. The relative mRNA expression levels were normalized to those of GAPDH, using the $2^{-\Delta \Delta C t}$ method (15). Primers are listed in Table I.

Western blot analysis. Following LDL treatment, the cells were lysed in lysis buffer (50 mm Tris, $\mathrm{pH} 7.4,150 \mathrm{~mm} \mathrm{NaCl}$, $1 \%$ NP-40, and $0.1 \%$ SDS), supplemented with protease 
inhibitors $(2 \mu \mathrm{g} / \mathrm{ml}$ aprotinin, $2 \mu \mathrm{g} / \mathrm{ml}$ leupeptin, and $1 \mathrm{~mm}$ phenylmethylsulfonyl fluoride). The protein samples were separated by electrophoresis on polyacrylamide gels containing $0.1 \%$ SDS, and then transferred to nitrocellulose membranes (Pierce Biotechnology, Inc., Rockford, IL, USA). The membranes were incubated with the individual primary antibodies (goat anti-mouse NF- $\mathrm{BB}$ p65 antibody, sc-166748; Santa Cruz Biotechnology, Inc; dilution, 1:1,000) overnight at $4^{\circ} \mathrm{C}$, followed by incubation for $1 \mathrm{~h}$ with the appropriate secondary antibodies (AlexaFluor ${ }^{\circledR}$ 488-labeled goat anti-rabbit immunoglobulin G, \#4412; Cell Signaling Technology, Inc.; dilution, 1:200), at room temperature. Immune complexes were visualized using enzyme-linked chemiluminescence (GE Heakthcare Life Sciences, Chalfont, UK). The band density was densitometrically analyzed using the Quantity One software (Bio-Rad Laboratories, Inc., Hercules, CA, USA), and normalized against the density of $\beta$-actin.

Immunocytochemistry staining. The cells were washed with phosphate-buffered saline (PBS; pH 7.4) and fixed in $4 \%$ formaldehyde in PBS for $15 \mathrm{~min}$. The cells were blocked by preincubation with 10\% normal goat serum in PBS for $1 \mathrm{~h}$ at room temperature, and were then incubated with anti-NF- $\mathrm{\kappa B}$ p65 antibodies, at $4^{\circ} \mathrm{C}$ overnight. Following further washes with PBS, the cells were incubated with Alexa Fluor ${ }^{\circledR}$ 488-labeled goat anti-rabbit immunoglobulin G (1:200 dilution), at room temperature for $1 \mathrm{~h}$. The cells were counterstained with 4,6-diamidino-2-phenylindole, in order to visualize the nuclei. The cells were visualized under a fluorescent microscope (LeicaTCS-SP5, Leica, Mannheim, Germany).

Statistical analysis. The data are expressed as the means \pm standard deviation. The statistical differences among the numerous groups were calculated using a one-way analysis of variance, followed by the Tukey post hoc test. A $\mathrm{P}<0.05$ was considered to indicate a statistically significant difference. All statistical tests were performed using SPSS version 13.0 software package for Windows (SPSS, Inc., Chicago, IL, USA)

\section{Results}

LDL treatment stimulates expression of CD40 and proinflammatory cytokines in HMCs. HMCs were exposed to LDL for various durations, and any changes to gene expression levels were determined by qPCR. LDL treatment caused a significant increase in the relative mRNA expression levels of IL-6, monocyte chemoattractant protein-1 (MCP-1), tumor necrosis factor- $\alpha$ (TNF- $\alpha$ ), and CD40, as compared with the untreated control cells $(\mathrm{P}<0.05$; Fig. 1). Furthermore, this induction was time-dependent, peaking at $24 \mathrm{~h}$ of culture.

Activation of the IREI $/ I K K / N F-\kappa B$ pathway is involved in LDL-induced proinflammatory cytokine expression. Treatment with LDL induced a time-dependent increase in the phosphorylation of IRE1 $\alpha$,IKK, and NF- $\mathrm{kB}$ p 65 , as determined by western blot analysis (Fig. 2A). This result is indicative of activation of the IRE1 $\alpha / \mathrm{IKK} / \mathrm{NF}-\kappa \mathrm{B}$ pathway. However, the total protein expression levels of IRE1 $\alpha$, IKK, and NF- $\kappa B$ p65 remained unchanged (data not shown). To further assess

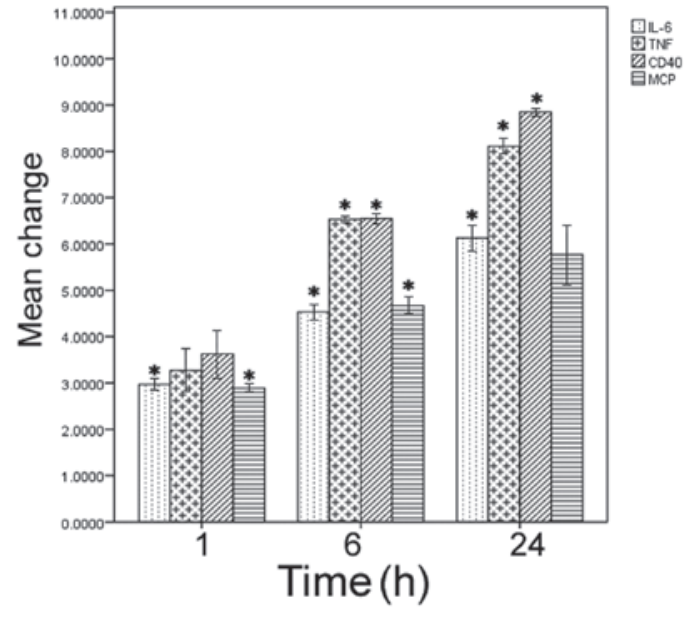

Figure 1. Quantitative polymerase chain reaction analysis of proinflammatory gene expression in human mesangial cells treated with low-density lipoprotein $(200 \mathrm{~nm})$, for the indicated durations. The results are expressed as the fold change, relative to the control cells $(0 \mathrm{~h})$, assigned as 1 -fold. ${ }^{*} \mathrm{P}<0.05$ vs. the control cells. H, hours; IL, interleukin; TNF, tumor necrosis factor; $\mathrm{MCP}$, monocyte chemoattractant protein.

the activation of NF- $\mathrm{BB}$ signaling, immunocytochemistry was used to examine the nuclear translocation of NF- $\mathrm{B}$ p65. Treatment with LDL markedly promoted NF- $\mathrm{KB}$ p65 nuclear translocation, in a time-dependent manner (Fig. 2B).

To confirm the essential role of IRE1 $\alpha$ in LDL-induced inflammatory responses, HMCs were transfected with IRE1 $\alpha$ specific siRNA, or scrambled control siRNA, $24 \mathrm{~h}$ before treatment with LDL. The delivery of the IRE1 $\alpha$ specific siRNA, but not control siRNA, markedly reduced the mRNA expression levels of endogenous IRE1 $\alpha$ in HMCs, as compared with the non-transfected cells (data not shown). Depletion of IRE1 $\alpha$ expression significantly blocked the induction of IL-6, MCP-1, TNF- $\alpha$, and CD40 exerted by LDL, as determined by qPCR $(\mathrm{P}<0.05$; Fig. 3A). Furthermore, knockdown of IRE1 $\alpha$ expression markedly reduced the phosphorylation levels of IKK and NF-kB p65 (Fig. 3B).

ER stress preconditioning attenuates the $L D L$-induced inflammatory response. The effects of ER stress preconditioning were also determined on LDL-induced inflammatory responses in HMCs. Pretreatment with tunicamycin significantly attenuated the induction of IL-6, MCP-1, TNF- $\alpha$, and CD40 by LDL ( $\mathrm{P}<0.05$; Fig. 4A). Furthermore, the phosphorylation levels of IRE1 $\alpha$, IKK, and NF- $\mathrm{BB}$ p65 were markedly reduced in the LDL-treated HMCs with tunicamycin pretreatment (Fig. 4B).

Tunicamycin pretreatment upregulates the expression levels of $X B P 1$ in HMCs. Treatment with tunicamycin alone markedly increased the protein expression levels of XBP1 in the HMCs, as determined by western blot analysis (Fig. 5A). However, the mRNA expression levels of IL-6, MCP-1, TNF- $\alpha$, and CD40 remained unchanged in the tunicamycin-treated cells (Fig. 5B).

Depletion of $X B P 1$ reverses the anti-inflammatory effects of ER stress preconditioning on LDL-treated HMCs. The role of XBP1 on the anti-inflammatory effects of ER stress preconditioning in HMCs was then determined. Transfection 


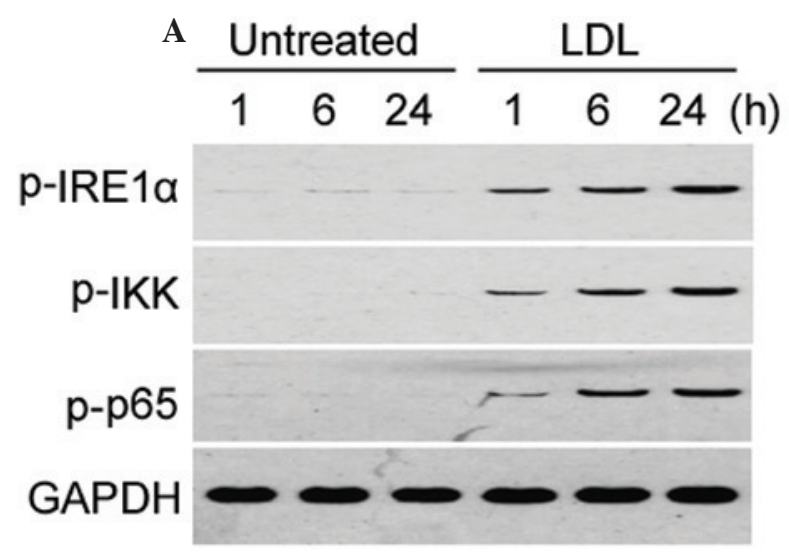

$\mathbf{B}$

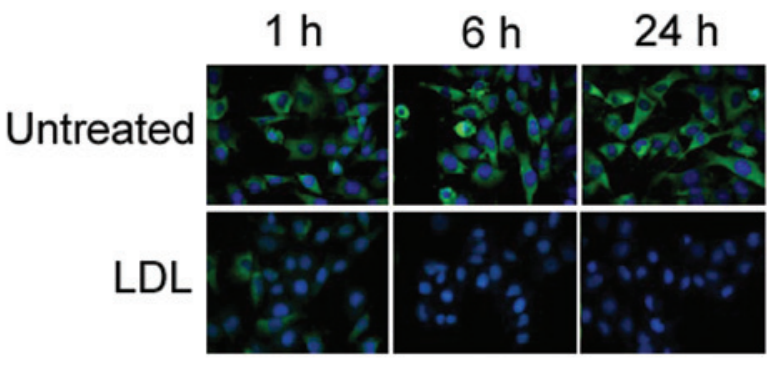

Figure 2. Treatment with low-density lipoprotein (LDL) activates the inositol-requiring enzyme (IRE) $1 \alpha /$ IKB kinase (IKK)/nuclear factor (NF)- $\mathrm{KB}$ pathway in human mesangial cells. (A) Western blot analysis of the phosphorylation of IRE1 $\alpha$, IKK, and NF-kB p65 in HMCs, with or without LDL treatment. The Western blots for LDL 1, 6, $24 \mathrm{~h}$ are presented again in Figs. 3B, 4B and 7B, and are used as a comparison for the other experimental conditions. Representative blots of three independent experiments with similar results are shown. (B) Immunohistochemistry for NF-אB p65 subcellular expression in HMCs, with or without LDL treatment. Green staining indicates the localization of p65, and blue staining indicates the nucleus. Magnification, x200; h, hours; p, phosphorylated.

A

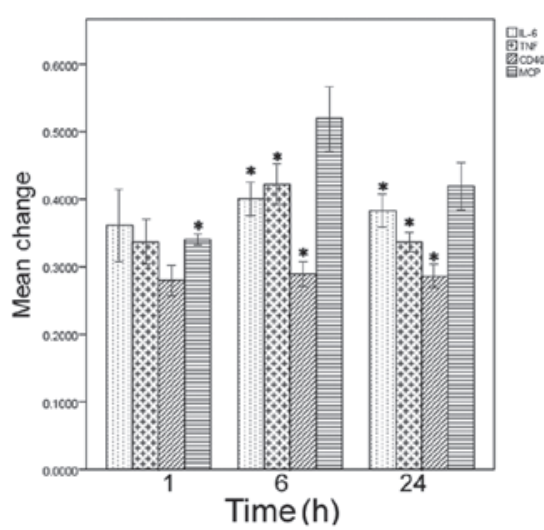

B

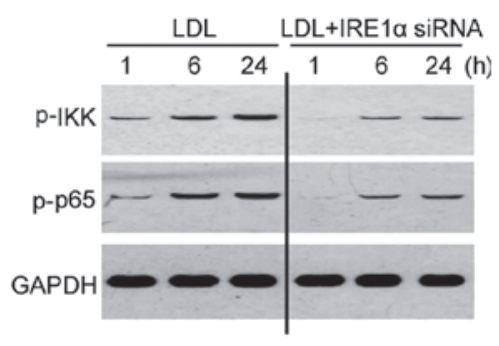

Figure 3. Inositol-requiring enzyme (IRE)1 $\alpha$ depletion blocks low-density lipoprotein (LDL)-induced inflammation in human mesangial cells (HMCs). The HMCs were transiently transfected with or without IRE1 $\alpha$ specific small interfering (si)RNA, $24 \mathrm{~h}$ prior to the addition of LDL, and then subjected to gene expression analysis. (A) Quantitative polymerase chain reaction analysis of mRNA expression levels of the indicated proinflammatory genes. The results are expressed as a fold change relative to the untreated cells, assigned as 1 -fold. "P $<0.05$ vs. the cells treated with LDL alone. (B) Western blot analysis of the

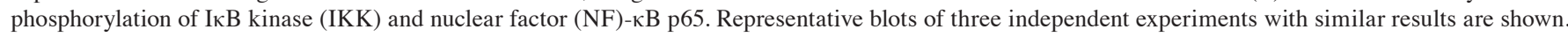
$\mathrm{H}$, hours; IL, interleukin; TNF, tumor necrosis factor; MCP, monocyte chemoattractant protein.

A

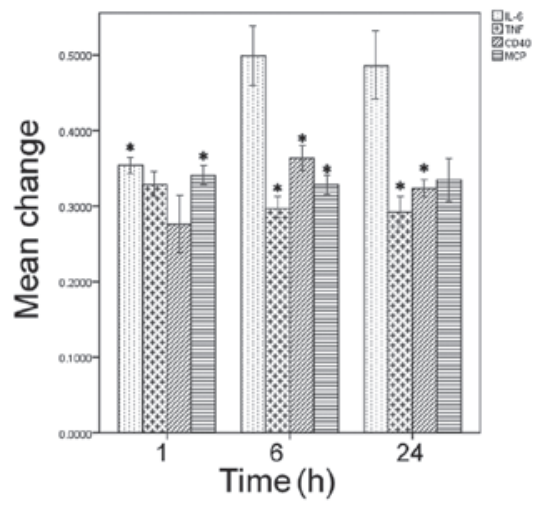

B

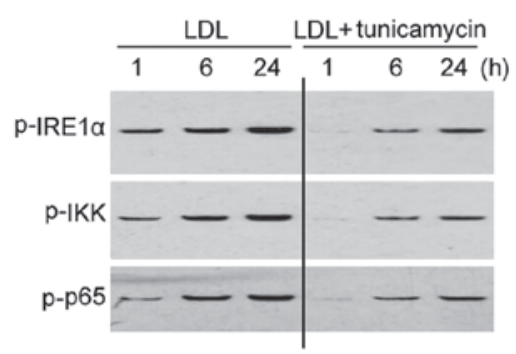

Figure 4. Endoplasmic reticulum (ER) stress preconditioning attenuates low-density lipoprotein (LDL)-induced inflammation in human mesangial cells (HMCs). The HMCs were exposed for $8 \mathrm{~h}$ to a low dose $(0.1 \mu \mathrm{g} / \mathrm{ml})$ of tunicamycin, prior to treatment with LDL. Following an incubation for $1-24 \mathrm{~h}$, the cells were tested for gene expression level changes. (A) Quantitative polymerase chain reaction analysis of mRNA expression levels of the indicated proinflammatory genes. The results are expressed as fold change relative to untreated cells, assigned as 1 -fold. ${ }^{*} \mathrm{P}<0.05$ vs. the cells treated with LDL alone. (B) Western blot analysis of the phosphorylation of inositol-requiring enzyme (IRE) $1 \alpha$, IкB kinase (IKK), and nuclear factor (NF)- $\kappa B$ p65. Representative blots of three independent experiments with similar results are shown. H, hours; IL, interleukin; TNF, tumor necrosis factor; MCP, monocyte chemoattractant protein. 
A

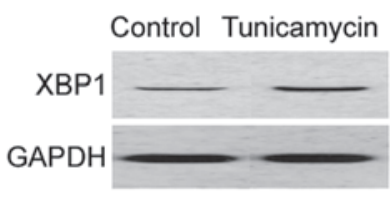

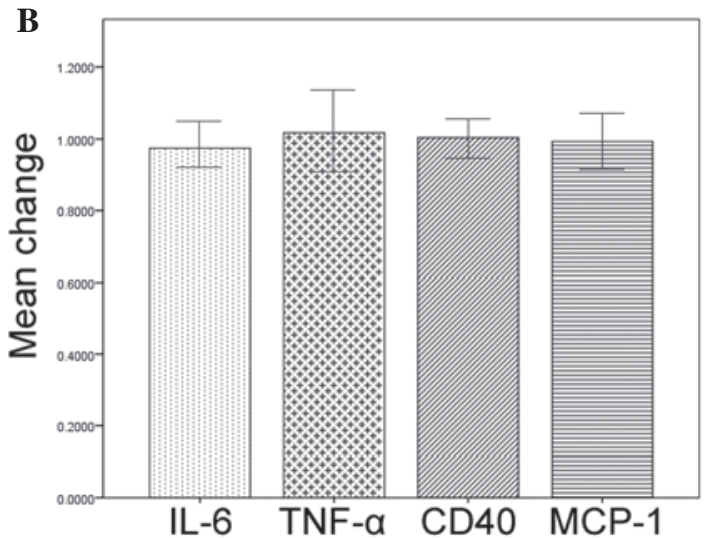

Figure 5. Tunicamycin treatment alone upregulates the expression levels of X-box-binding protein-1 (XBP1) in human mesangial cells (HMCs). The HMCs were treated with tunicamycin $(0.1 \mu \mathrm{g} / \mathrm{ml})$ for $8 \mathrm{~h}$, and a gene expression analysis was conducted. (A) Western blot analysis of XBP1 protein expression levels. A representative blot of three independent experiments with similar results is shown. (B) Quantitative polymerase chain reaction analysis of mRNA expression levels of the indicated proinflammatory genes. The results are expressed as fold change relative to untreated cells, assigned as 1 -fold. ${ }^{*} \mathrm{P}<0.05$ vs. the untreated cells. H, hours; IL, interleukin; TNF, tumor necrosis factor; MCP, monocyte chemoattractant protein.

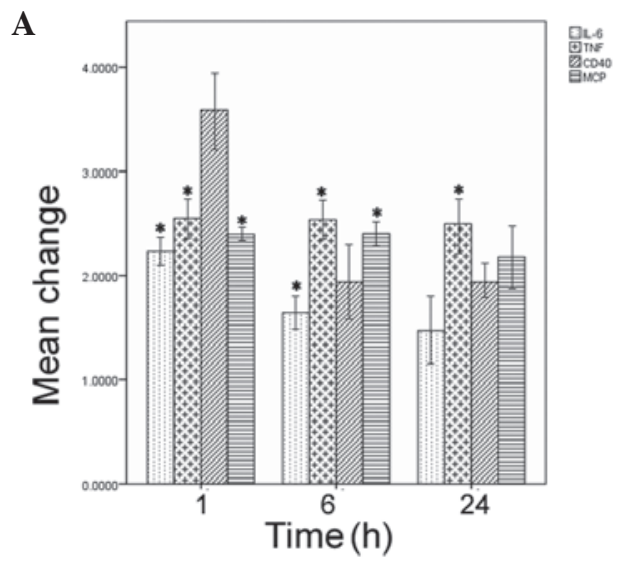

B

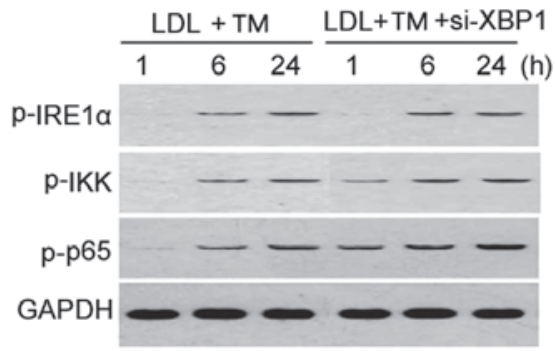

Figure 6. Silencing of X-box-binding protein-1 (XBP1) expression reverses the anti-inflammatory effects of tunicamycin pretreatment on low-density lipoprotein (LDL)-treated human mesangial cells (HMCs). The HMCs were transiently transfected with or without XBP1 specific small interfering (si)RNA, $24 \mathrm{~h}$ prior to treatment with tunicamycin (TM) and LDL and then subjected to gene expression analysis. (A) Quantitative polymerase chain reaction analysis of the mRNA expression levels of the indicated proinflammatory genes. The results are expressed as a fold change relative to the untreated cells, assigned as 1-fold. ${ }^{*} \mathrm{P}<0.05$ vs. cells treated with LDL alone. (B) Western blot analysis of the phosphorylation of. Representative blots of three independent experiments with similar results are shown. H, hours; IL, interleukin; TNF, tumor necrosis factor; MCP, monocyte chemoattractant protein; IRE1 $\alpha$, inositol-requiring enzyme; IKK, IкB kinase; p65, nuclear factor-кB p65.

A

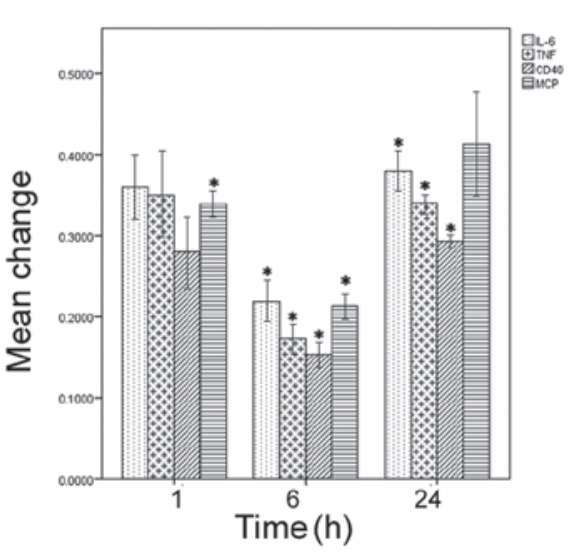

B

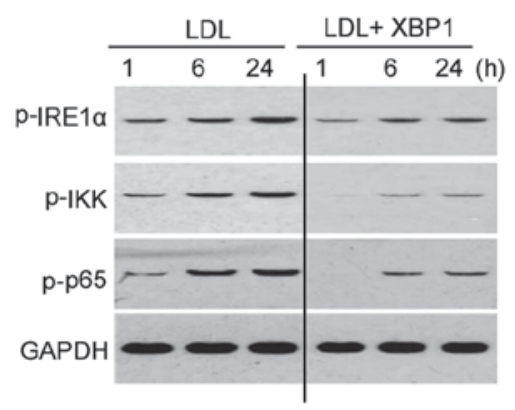

Figure 7. Overexpression of X-box-binding protein-1 (XBP1) diminishes low-density lipoprotein (LDL)-induced inflammation in human mesangial cells (HMCs). The HMCs were transiently transfected with or without XBP1-expressing plasmid, $24 \mathrm{~h}$ prior to treatment with LDL, and then subjected to gene expression analysis. (A) Quantitative polymerase chain reaction analysis of mRNA expression levels of the indicated proinflammatory genes. The results are expressed as a fold change relative to the untreated cells, assigned as 1 -fold. " $\mathrm{P}<0.05$ vs. the cells treated with LDL alone. (B) Western blot analysis of the phosphorylation of inositol-requiring enzyme (IRE) $1 \alpha$, IкB kinase (IKK), and nuclear factor (NF)- $\mathrm{kB}$ p65. Representative blots of three independent experiments with similar results are shown. H, hours; IL, interleukin; TNF, tumor necrosis factor; MCP, monocyte chemoattractant protein. 
with specific XBP1 siRNA resulted in a marked reduction in endogenous XBP1 protein expression levels in the HMCs, as compared with the cells transfected with control siRNA (data not shown). Notably, silencing XBP1 expression significantly restored the mRNA expression of IL-6, MCP-1, TNF- $\alpha$, and CD40, in the LDL-treated HMCs with ER stress preconditioning $(\mathrm{P}<0.05$; Fig. $6 \mathrm{~A})$. Furthermore, the phosphorylation levels of IRE1 $\alpha$, IKK, and NF- $\kappa$ B p 65 were markedly increased in the XBP1-depleted HMCs with ER stress preconditioning (Fig. 6B).

Overexpression of XBP1 antagonizes $L D L$-induced inflammation. The present study also examined whether overexpression of XBP1 could block LDL-induced inflammatory responses in HMCs. The overexpression of XBP1 in the HMCs transfected with the XBP1-expressing plasmid was confirmed by western blot analysis (data not shown). Overexpression of XBP1 significantly reduced the mRNA expression levels of IL-6, MCP-1, TNF- $\alpha$, and CD40 in LDL-treated HMCs, as determined by qPCR (Fig. 7A). Furthermore, the phosphorylation levels of IRE1 $\alpha$, IKK, and NF- $\kappa \mathrm{B}$ p65 in LDL-treated cells were diminished in response to overexpression of XBP1 (Fig. 7B).

\section{Discussion}

Native and modified LDL has previously been shown to possess potent proinflammatory activities in various biological contexts. Meng et al (16) reported that oxidized LDL triggers inflammatory responses in cultured human mast cells by activating the Toll-like receptor 4-dependent signaling pathway. Oxidized LDL has also been shown to stimulate proinflammatory responses in alternatively activated M2 macrophages (17). The present study showed that LDL treatment promoted inflammatory responses in HMCs, as evidenced by the increased expression levels of proinflammatory cytokines IL-6, MCP-1, and TNF- $\alpha$. These results are concordant with previous studies $(14,18)$. Systemic inflammation is regarded as a key factor in the pathogenesis of CKD (13). The proinflammatory activity of LDL in HMCs may partially explain the association between dyslipidemia and the progression of CKD (2).

CD40 is expressed in a wide range of cells, including macrophages, lymphocytes, endothelial cells, vascular smooth muscle cells, and mesangial cells $(19,20)$. CD40/CD40 ligand interactions between infiltrating mononuclear cells and resident renal cells, are associated with the pathogenesis of immune-mediated glomerulonephritis (21). Increasing evidence has indicated that activation of the CD40/CD40 ligand pathway is associated with the initiation of inflammatory responses (19). Tanaka et al (22) reported that human platelets stimulate mesangial cells to produce MCP-1, via the CD40/CD40 ligand pathway. The results of the present study demonstrated that upregulation of CD40 expression occurs in LDL-induced inflammatory responses in HMCs. In addition to initiation of inflammatory responses, activation of CD40-dependent signaling has been found to protect HMCs from oxidized LDL-induced apoptosis (23). These findings suggest that LDL-mediated toxic effects on HMCs are associated with the regulation of CD40 signaling.

There is known to be a close association between ER stress and the inflammatory response (24). Li et al (25) showed that induction of ER stress contributes to retinal inflammation in diabetic retinopathy (24). The results of the present study showed that, in parallel with induction of proinflammatory cytokines, LDL treatment resulted in a time-dependent increase in the phosphorylation of IRE1 $\alpha$, IKK, and NF- $\kappa$ B p65. IRE1 $\alpha$ is a major sensor of unfolded proteins in the ER, and is activated by autophosphorylation (7). In response to ER stress, activated IRE1 $\alpha$ may bind to the IKK complex and activate $\mathrm{NF}-\kappa \mathrm{B}(10,26)$. In the present study siRNA technology was used to induce a specific depletion of IRE1 $\alpha$ expression. The loss of IRE1 $\alpha$ expression antagonized the induction of IL-6, MCP-1, TNF- $\alpha$, and CD40 exerted by LDL, coupled with the suppression of $\mathrm{NF}-\kappa \mathrm{B}$ activation. These findings indicate that ER stress has a mediating role in LDL-induced inflammatory responses in HMCs, which involves the activation of the IRE1 $\alpha / \mathrm{IKK} / \mathrm{NF}-\kappa \mathrm{B}$ pathway. Consistently, phospholipolyzed LDL has been found to induce an inflammatory response in endothelial cells, by activating ER stress signaling pathways (27).

Accumulating evidence indicates that ER stress preconditioning confers protection against cellular injury in various biological settings $(12,28)$. Hung et al $(28)$ reported that ER stress preconditioning attenuated hydrogen peroxide-induced oxidative injury in LLC-PK1 renal epithelial cells. Li et al (10) previously demonstrated that preconditioning with ER stress mitigated retinal endothelial inflammation. The present study showed that LDL-induced inflammatory cytokine production was significantly diminished in ER stress-primed HMCs, suggesting a protective role for ER stress preconditioning. Notably, the results of the present study revealed that pretreatment with the ER stress inducer tunicamycin, caused an upregulation of XBP1, but not of the proinflammatory cytokines. In addition, siRNA-mediated inhibition of XBP1 attenuated the protective effects of tunicamycin pretreatment on LDL-induced inflammation, which was coupled with an enhanced activation of the IRE1 $\alpha / \mathrm{IKK} / \mathrm{NF}-\kappa \mathrm{B}$ pathway. Numerous studies have shown that XBP1 ablation triggers feedback activation of its upstream enzyme IRE1 $\alpha(29,30)$. Furthermore, the present study revealed that XBP1 overexpression inhibited LDL-induced activation of the IRE1 $\alpha / \mathrm{IKK} / \mathrm{NF}-\kappa \mathrm{B}$ pathway. These results collectively indicate that ER stress preconditioning ameliorates LDL-induced inflammation in HMCs, which is largely mediated through activation of XBP1 signaling and blockade of the IRE1 $\alpha / \mathrm{IKK} / \mathrm{NF}-\kappa \mathrm{B}$ pathway.

In conclusion, activation of the IRE $\alpha / \mathrm{IKK} / \mathrm{NF}-\kappa \mathrm{B}$ pathway is required for LDL-induced inflammation in HMCs. ER stress preconditioning resulted in the upregulation of XBP1 expression and subsequent inhibition of the IRE1 $\alpha / \mathrm{IKK} / \mathrm{NF}-\kappa \mathrm{B}$ pathway, which consequently interfered with the LDL-induced inflammatory responses. These findings warrant further investigation of the therapeutic potential of ER stress preconditioning in inflammatory disorders, such as CKD.

\section{References}

1. Wakasugi M, Kazama JJ and Narita I: Differences in the local and national prevalences of chronic kidney disease based on annual health check program data. Clin Exp Nephrol 16: 749-754, 2012.

2. Nitta K: Clinical assessment and management of dyslipidemia in patients with chronic kidney disease. Clin Exp Nephrol 16: 522-529, 2012. 
3. Vaziri ND and Norris K: Lipid disorders and their relevance to outcomes in chronic kidney disease. Blood Purif 31: 189-196, 2011.

4. Podrez EA: Anti-oxidant properties of high-density lipoprotein and atherosclerosis. Clin Exp Pharmacol Physiol 37: 719-725, 2010.

5. Pirillo A, Norata GD and Catapano AL: LOX-1, OxLDL, and atherosclerosis. Mediators Inflamm 2013: 152786, 2013.

6. Xu C, Bailly-Maitre B and Reed JC: Endoplasmic reticulum stress: cell life and death decisions. J Clin Invest 115: 2656-2664 2005.

7. Inagi R: Endoplasmic reticulum stress as a progression factor for kidney injury. Curr Opin Pharmacol 10: 156-165, 2010.

8. Dickhout JG and Krepinsky JC: Endoplasmic reticulum stress and renal disease. Antioxid Redox Signal 11: 2341-2352, 2009.

9. Chiang CK, Hsu SP, Wu CT, et al: Endoplasmic reticulum stress implicated in the development of renal fibrosis. Mol Med 17: 1295-1305, 2011.

10. Li J, Wang JJ and Zhang SX: Preconditioning with endoplasmic reticulum stress mitigates retinal endothelial inflammation via activation of X-box binding protein 1. J Biol Chem 286: 4912-4921, 2011

11. Aminzadeh MA, Sato T and Vaziri ND: Participation of endoplasmic reticulum stress in the pathogenesis of spontaneous glomerulosclerosis - role of intra-renal angiotensin system. Transl Res 160: 309-318, 2012.

12. Inagi R, Kumagai T, Nishi H, et al: Preconditioning with endoplasmic reticulum stress ameliorates mesangioproliferative glomerulonephritis. J Am Soc Nephrol 19: 915-922, 2008.

13. Cottone $\mathrm{S}$, Lorito MC, Riccobene $\mathrm{R}$, et al: Oxidative stress, inflammation and cardiovascular disease in chronic renal failure. J Nephrol 21: 175-179, 2008.

14. Santini E, Lupi R, Baldi S, et al: Effects of different LDL particles on inflammatory molecules in human mesangial cells Diabetologia 51: 2117-2125, 2008

15. Livak MJ and Schmittgen TD: Analysis of relative gene expression data using real-time quantitative PCR and the 2(-Delta Delta C(T)) method. Methods 25: 402-408, 2001.

16. Meng Z, Yan C, Deng Q, et al: Oxidized low-density lipoprotein induces inflammatory responses in cultured human mast cells via Toll-like receptor 4. Cell Physiol Biochem 31: 842-853, 2013.

17. van Tits LJ, Stienstra R, van Lent PL, et al: Oxidized LDL enhances pro-inflammatory responses of alternatively activated M2 macrophages: a crucial role for Krüppel-like factor 2. Atherosclerosis 214: 345-349, 2011.

18. Massy ZA, Kim Y, Guijarro C, et al: Low-density lipoprotein-induced expression of interleukin-6, a marker of human mesangial cell inflammation: effects of oxidation and modulation by lovastatin. Biochem Biophys Res Commun 267: 536-540, 2000.
19. Antoniades C, Bakogiannis C, Tousoulis D, Antonopoulos AS and Stefanadis C: The CD40/CD40 ligand system: linking inflammation with atherothrombosis. J Am Coll Cardiol 54: 669-677, 2009

20. Yellin MJ, D'Agati V, Parkinson G, et al: Immunohistologic analysis of renal CD40 and CD40L expression in lupus nephritis and other glomerulonephritides. Arthritis Rheum 40: 124-134, 1997.

21. Kuroiwa T, Schlimgen R, Illei GG, McInnes IB and Boumpas DT: Distinct T cell/renal tubular epithelial cell interactions define differential chemokine production: implications for tubulointerstitial injury in chronic glomerulonephritides. J Immunol 164: 3323-3329, 2000

22. Tanaka T, Kuroiwa T, Ikeuchi H, et al: Human platelets stimulate mesangial cells to produce monocyte chemoattractant protein-1 via the CD40/CD40 ligand pathway and may amplify glomerular injury. J Am Soc Nephrol 13: 2488-2496, 2002.

23. Deambrosis I, Scalabrino E, Deregibus MC, Camussi G and Bussolati B: CD40-dependent activation of phosphatidylinositol 3-kinase/AKT pathway inhibits apoptosis of human cultured mesangial cells induced by oxidized LDL. Int J Immunopathol Pharmacol 18: 327-337, 2005.

24. Su J, Zhou L, Kong X, et al: Endoplasmic reticulum is at the crossroads of autophagy, inflammation, and apoptosis signaling pathways and participates in the pathogenesis of diabetes mellitus. J Diabetes Res 2013: 193461, 2013.

25. Li J, Wang JJ, Yu Q, Wang $M$ and Zhang SX: Endoplasmic reticulum stress is implicated in retinal inflammation and diabetic retinopathy. FEBS Lett 583: 1521-1527, 2009.

26. Hayakawa K, Hiramatsu N, Okamura M, et al: Acquisition of anergy to proinflammatory cytokines in nonimmune cells through endoplasmic reticulum stress response: a mechanism for subsidence of inflammation. J Immunol 182: 1182-1191, 2009.

27. Gora S, Maouche S, Atout R, et al: Phospholipolyzed LDL induces an inflammatory response in endothelial cells through endoplasmic reticulum stress signaling. FASEB J 24: 3284-3297, 2010.

28. Hung CC, Ichimura T, Stevens JL and Bonventre JV: Protection of renal epithelial cells against oxidative injury by endoplasmic reticulum stress preconditioning is mediated by ERK1/2 activation. J Biol Chem 278: 29317-29326, 2003.

29. So JS, Hur KY, Tarrio M, et al: Silencing of lipid metabolism genes through IRE1 $\alpha$-mediated mRNA decay lowers plasma lipids in mice. Cell Metab 16: 487-499, 2012.

30. Lee AH, Heidtman K, Hotamisligil GS and Glimcher LH: Dual and opposing roles of the unfolded protein response regulated by IRE1alpha and XBP1 in proinsulin processing and insulin secretion. Proc Natl Acad Sci USA 108: 8885-8890, 2011 